\title{
Place Name
}

National Cancer Institute

\section{Source}

National Cancer Institute. Place Name. NCI Thesaurus. Code C95378.

The literal identifier of the physical place. 